\title{
Linear Tapered Slot Antennas on LTCC Substrate for Millimeter-wave Applications
}

\author{
Il Kwon Kim, Stephan Pinel*, John Papapolymerou*, \\ Manos M. Tentzeris*, Joy Laskar*, and Jong-Gwan Yook \\ Dept. of Electrical and Electronic Engineering, Yonsei University \\ 134, Shinchon-Dong, Seodaemum-Gu, Seoul, Korea, 120-749 \\ *School of Electrical and Computer Engineering, Georgia Institute of Technology \\ Atlanta, GA 30332-0250, USA \\ kimik@yonsei.ac.kr
}

Abstract- In this paper, one linear tapered slot antenna (LTSA), backed with an air cavity, is proposed and fabricated on low temperature cofired ceramic (LTCC) substrate. Conventionally, when LTSA is designed on LTCC board, due to its high dielectric constant and thickness, the gain and radiation property is degraded seriously. Therefore in this paper, by adopting air cavity in the back side of LTCC substrate, high gain and good endfire radiation pattern can be obtained. Especially, this antenna has broad bandwidth (about from $45 \mathrm{GHz}$ to $75 \mathrm{GHz}$ ) and high gain $(4.9$ - $6.9 \mathrm{~dB}$ ) property. This antenna can be used for a variety of millimeter-wave applications, due to its easy integrability with $3 \mathrm{D}$ LTCC modules.

\section{Introduction}

In these days, millimeter-wave electronics for commercial applications, such as shortrange broadband wireless communications, automotive collision avoidance radars and local cellular radio network (LCRN) require low fabrication cost, excellent performance, and high level of integration. To satisfy these properties, low temperature cofired ceramic (LTCC) technology has been studied by many researchers. [1][2] However, in the view point of the antenna, the high dielectric constant of the LTCC material causes serious problems to the design of antennas with high gain and good radiation pattern, especially, for endfire antenna. There are several types of the end-fire case, such as horn waveguide antenna, Yagi antenna and linear tapered slot antenna (LTSA). Among them, the linear tapered slot antenna [3]-[5] is a good candidate for LTCC design, for reasons such as easy fabrication, broad bandwidth, and easy integration. There are numerous variations of LTSA, such as Vivaldi, exponential and constant width, depending on the aperture shape of antenna. All of these types aim to improve beam width property, broaden the bandwidth, and achieve good matching [6].

In this paper, LTSA is designed and fabricated by using LTCC process for millimeterwave applications. Usually, antennas on high dielectric substrate have problems, such as lower gain and distorted radiation pattern due to high quality factor of antenna. To alleviate this effect, several methods have been proposed to lower the high dielectric constant, such as material modulation [7][8] or photonic band gap (PBG) [9]. Due to the high dielectric constant and thickness of LTCC (the permittivity 5.4 and thickness 900 um), an air cavity is used to achieve broadband performance and lower the effective dielectric constant. The effective thickness for good performance is given as $0.005 \lambda_{0} \leq$ effective thickness $\leq 0.03 \lambda_{0}[7]$.

\section{II.LTSA with air cavity back}

In this paper, an LTSA backed with an air cavity is proposed and its characteristics are investigated by using numerical and experimental method. All LTCC boards consist of 9 layers and the thickness of each layer (the permittivity 5.4) is $100 \mathrm{um}$. Therefore, the total 
thickness of this LTCC board is $900 \mathrm{um}$. The proposed antenna is consisted by 3 major parts, radiation, feeding structure, and air-cavity as shown in Fig.1. The radiation part is located in $2^{\text {nd }}$ layer and its shape is linear type slot. The feeding structure is a microstrip to slotline transition and no additional matching circuit is required. An air cavity is located on the bottom layers of the LTCC board and the physical size is $5 \mathrm{~mm} \times 2.5 \mathrm{~mm}$. All configuration and physical dimensions of structure also can be shown in Fig.1 (a).

\section{Simulation and Experimental Results}

The characteristics of proposed antenna are investigated by using numerical tool, HFSS and experiments. Fig.1 (b) shows the fabricated antenna and Fig.2. displays the return loss performance of the antenna from $35 \mathrm{GHz}$ to $85 \mathrm{GHz}$. It can be seen that the $\mathrm{S} 11$ bandwidth of this antenna is $32 \mathrm{GHz}(43 \mathrm{GHz}-75 \mathrm{GHz})$ for VSWR 2:1. The simulation and measurement results show good agreement. Although, the impedance bandwidth is wider than $32 \mathrm{GHz}$ as shown in Fig.3, the effective radiation bandwidth is approximately $30 \mathrm{GHz}$, because the radiation pattern is distorted at frequencies higher than $75 \mathrm{GHz}$. Fig. 3 shows the simulated radiation pattern of the proposed antenna for $45 \mathrm{GHz}$ and 70 GHz. These 2D patterns are XY cut (azimuth plane) and demonstrate that this antenna has a satisfactory end-fire pattern property throughout the whole frequency range of operation. Additionally, the gain of this antenna ranges from $4.9 \mathrm{~dB}$ at the edges $(45 \mathrm{GHz}$ and $75 \mathrm{GHz}$ ) to $6.9 \mathrm{~dB}$ around the center frequency of $60 \mathrm{GHz}$.

\section{Conclusions}

In this paper, an LTSA backed with air cavity is proposed in LTCC technology and its characteristics are investigated up to $85 \mathrm{GHz}$. From simulation and measurement results, it is found that the proposed antenna has a broad bandwidth $(>30 \mathrm{GHz})$ and efficient end fire radiation pattern. Therefore this antenna can be used for a variety of millimeter wave applications, since it can be easily integrated with 3D LTCC modules.

\section{References}

[1] Kyutae lim, Stephane Pinel, Mekita Davis, Albert Sutono, Chang-Ho Lee, Deukhyoun Heo, Ade Obtoynbo, Joy Laskar, Emmanouli M. Tentzeris, and Rao Tummala, "RF System-OnPackage (SOP) for Wireless Communications," IEEE microwave magazine, pp.88-pp.99, Mar. 2002.

[2] Rao R. Tummala, "SOP: What Is It and Why? A New Microsystem-Integration Technology Paradigm-Moore's Law for System Integration of Miniaturized Convergent Systems of the Next Decade," IEEE Trans. on Advanced Packaging, vol.27, no.2, pp.241-pp.249, May 2004.

[3] P.J. Gibson, "The Vivaldi Aerial," in Proc. 9 $9^{\text {th }}$ Eur. Microwave Conf., pp. 101-105, June 1979, Brighton, UK

[4] K.S. Yngvesson, "Endfire tapered slot antennas on dielectric substrates," IEEE Trans. Antennas Propogat., vol.33, pp. 1392-1400, Dec. 1985.

[5] K. S. Yngvesson, T.L. Korzeniowski, Y.S. Kim, E.L. Kollberg, and J. F. Johansson, "The tapered slot antenna-A new integrated element for millimeter-wave applications,'IEEE Trans. Microwave Theory Tech., vol.37, pp.365-374, Feb.1989.

[6] K. Sigfirid Yngvesson, Daniel H. Schaubert, Thomas L. Korzeniowski, Erik L. Kollberg, Tomas Thungren, and Joakim F. Johansson, “ Endfire Tapred Slot Antennas on Dielectric Substrates," IEEE Trans. on Antennas and Propagation, vol.AP-33, no.12, pp.1392-1400, Dec. 1985.

[7] Jeremy B. Muldavin and Gabriel M. Rebeiz, "Millimeter-Wave Tapered Slot Antenna on Synthesized Low Permittivity Substrates," IEEE Trans. on Antennas and Propagation, vol.47, no.8, pp.1276-1280, Aug. 1999.

[8] Karsin Schuler, Yan Venot, and Werner Wiesbeck, "Innovative Material Modulation for Multilayer LTCC Antenna Design at 76.5 GHz in Radar and Communication Application," $33^{\text {rd }}$ European Microwave Conference, pp.707-pp.710, Oct. 2003. Munich. 
[9] Thomas J. Ellis and Gabriel M. Rebeiz, "MM-Wave Tapered Slot Antennas on Micromachined Photonic Bandgap Dielectrics," IEEE MTT-S Digest, pp.1157-pp.1160, 1996, San Francisco.
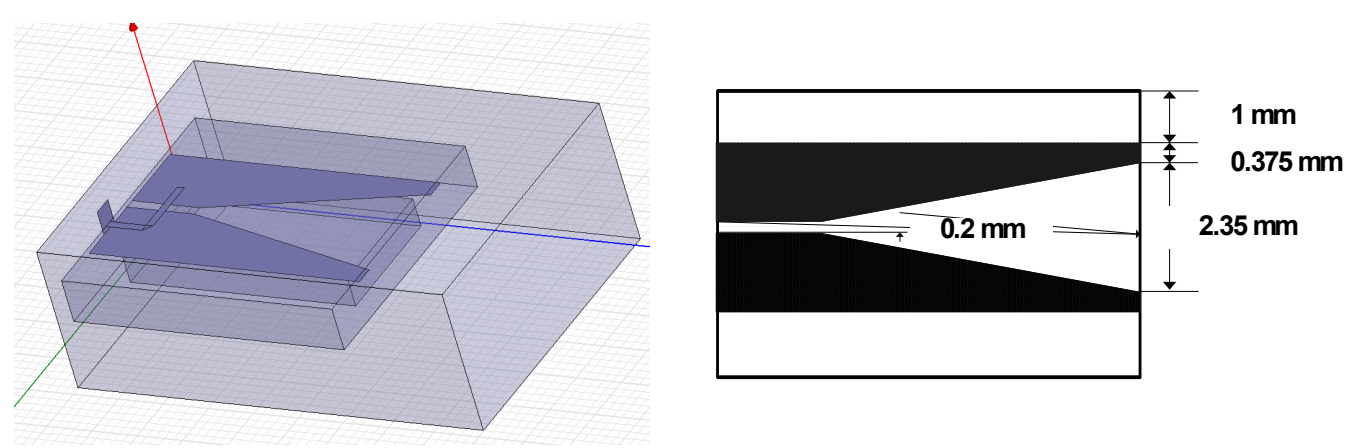

(a) Antenna configuration and slot antenna layout
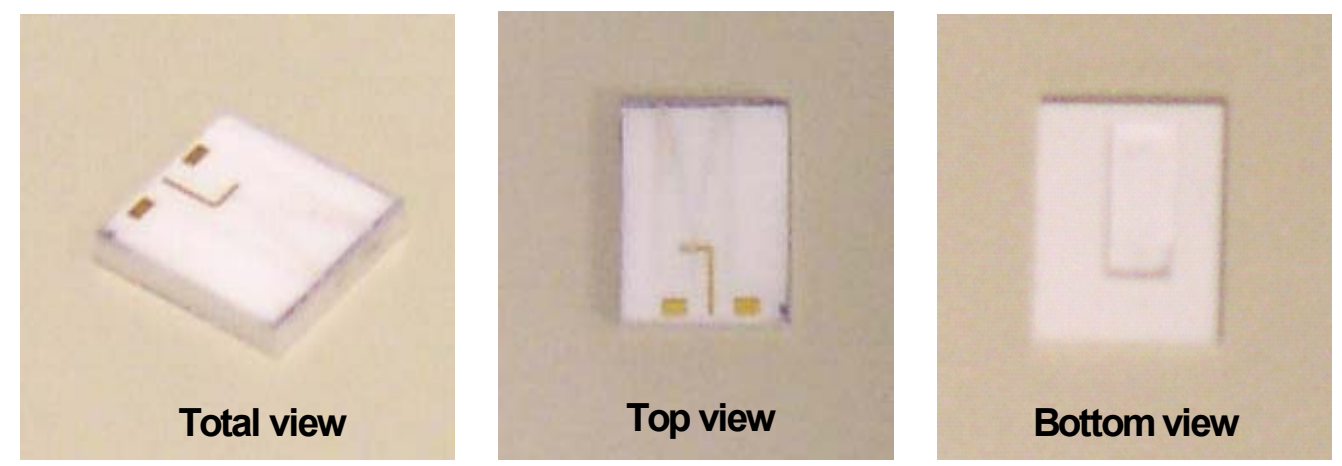

(b) Fabricated Antenna

Fig.1. Simulation Schematic and Fabricated antenna

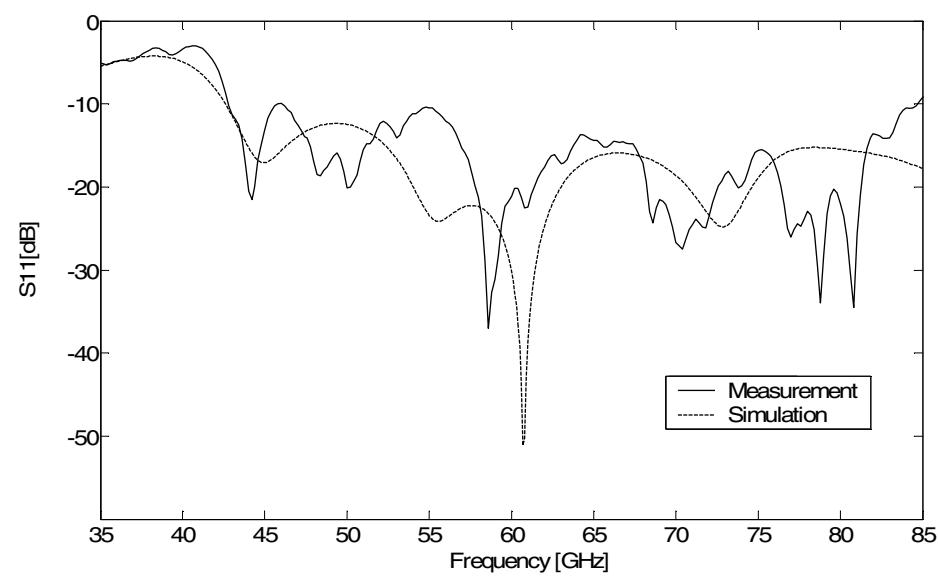

Fig.2. Return loss of proposed antenna 


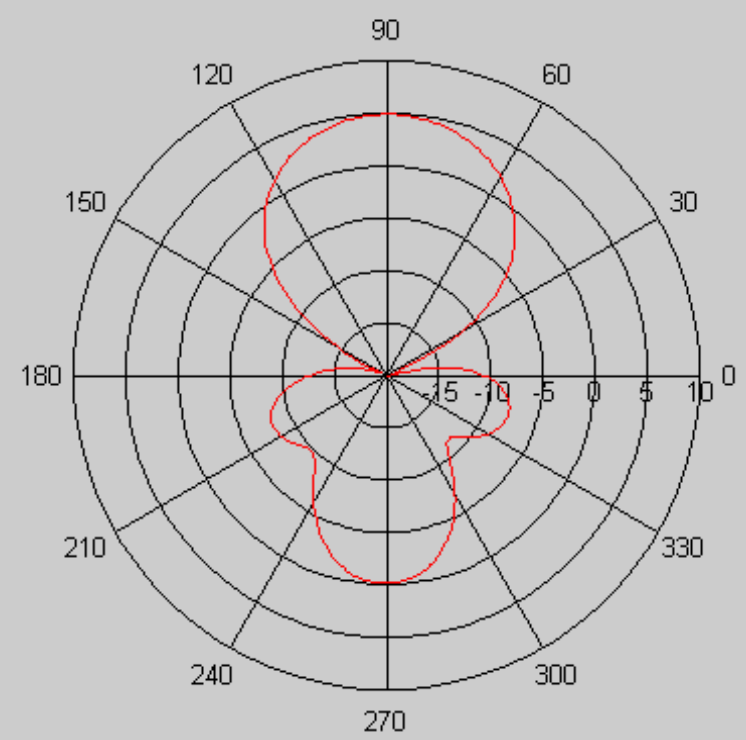

$45 \mathrm{GHz}$ (Gain $4.9 \mathrm{~dB}$ )

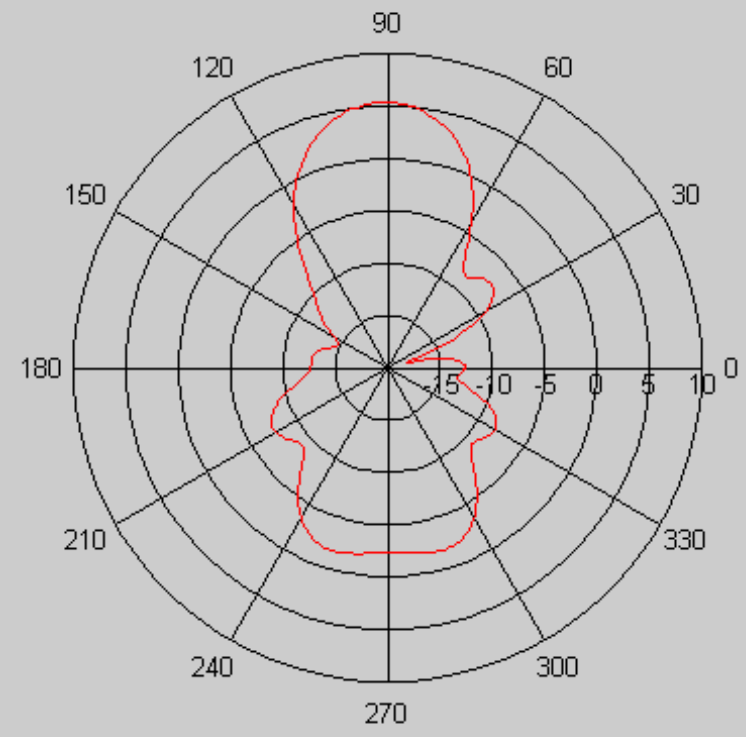

$70 \mathrm{GHz}$ (Gain $5.9 \mathrm{~dB}$ )

Fig.3. Radiation pattern and the gain (XY cut: Azimuth plane) 